\title{
Therapeutic potential of carbonyl-scavenging carnosine derivative in metabolic disorders
}

\author{
Jacob M. Haus ${ }^{1}$ and John P. Thyfault ${ }^{2}$ \\ ${ }^{1}$ School of Kinesiology, University of Michigan, Ann Arbor, Michigan, USA. Department of Molecular and Integrative Physiology, University of Kansas Medical School, Kansas City, Kansas, USA.
}

\begin{abstract}
Obesity and overnutrition increase levels of reactive sugar- and lipid-derived aldehydes called reactive carbonyl species (RCS). Increased tissue and circulating RCS levels have been tied to insulin resistance and inflammation, but previous pharmacological approaches to target RCS have had equivocal outcomes. In this issue of the $J C l$, Anderson et al. present evidence for the development and implementation of carnisonol, a compound that is biologically stable in vivo and shows impressive effects on improving metabolism and inflammation in rodent models of diet-induced obesity and metabolic dysfunction.
\end{abstract}

\section{Introduction}

Studies examining the mechanisms that drive insulin resistance and hyperglycemia have been a particular area of focus because of the rising rates of type 2 diabetes. Accumulating evidence shows a variety of potential mechanisms including ectopic lipid storage, lipid intermediates, inflammation, and mitochondrial dysfunction that may play roles in driving insulin resistance and hyperglycemia. Additionally, oxidative stress is also believed to play an important role. Intracellular and circulating markers of oxidative stress have been shown to track with worsening glycemia, insulin resistance, and lipid profiles, while weight loss and exercise interventions which improve these outcome measures also lower markers of oxidative stress. Oxidatives stress remains on the "outside looking in," because there is still a lack of direct mechanistic evidence elucidating its role in the induction of insulin resistance. A lack of firm evidence is likely due to the multiple types of oxidative stress due identified through various outcome measures in different tissues or in the circulation, the transient nature of oxidative reactions, the difficulty in assessing quality outcome measures and, most important, the small number of studies directly targeting specific types of oxidative stress to assess improvement in outcomes.

Reactive sugar- and lipid-derived aldehydes called reactive carbonyl species (RCS) are spontaneously formed during food preparation under high heat and as by-products of oxidative stress. These RCS damage proteins via the covalent modification of arginine, cystine, histidine, and lysine residues, as well as through the formation of adducts with DNA and phospholipids. A positive energy balance at the cellular level from an oversupply of either lipids or carbohydrates promotes oxidative stress and leads to an increased formation of RCS-modified proteins. In particular, the oxidation of $n-6$ polyunsaturated fatty acids (PUFAs) leads to the formation of the $\alpha, \beta$-unsaturated carbonyl species hydroxynonenal (HNE) and acrolein (ACR), each of which have been highly studied because they react readily with cellular proteins. HNE tracks with worsening obesity and metabolic dysfunction, and there is some evidence to suggest that these protein modifications underlie mechanisms of insulin resistance, fibrosis, and elevated lipids. Since the original characterization of HNE by Esterbauer et al. (1), the subsequent research involving this

Related Article: p. 5280

Conflict of interest: The authors have declared that no conflict of interest exists.

Reference information: J Clin Invest. 2018;128(12):5198-5200. https://doi.org/10.1172/JCI124304.

chemical has led to progress in numerous neutralization strategies.

The natural defense against HNEmediated carbonyl stress is detoxification through a combination of aldehyde dehydrogenases and a family of glutathione S-transferase enzymes that create a stable, water-soluble glutathione S-hydroxynonenal (GS-HNE) that is shuttled out of the cell (Figure 1). Importantly, L-carnosine, a naturally occurring dipeptide, also has the ability to scavenge intracellular RCS, forming unreactive covalent adducts (2, 3 ) which are then excreted in the urine (4), thus providing an additional mechanism of RCS detoxification. Naturally occurring L-carnosine has been shown to be reduced in obesity, leading to a greater susceptibility to RCS accumulation. L-carnosine supplementation has been effective in lowering RCS in rodent models; however, only a few studies have been performed in humans (2, 4-9). Recently, two separate carnosine supplementation interventions ( $2 \mathrm{~g} / \mathrm{d}$ for $12 \mathrm{wk}$ ) were performed in overweight-obese subjects. De Courten and colleagues found that two-hour glucose and insulin levels after a 75-g oral glucose tolerance test were both lower after carnosine supplementation compared with placebo in individuals with impaired glucose tolerance (7). On the other hand, Regazonni and colleagues found that carnosine bioavailability was limited because of rapid degradation by the carnosinase enzyme in tissues and serum, and carnosine-ACR adducts were detected in the urine of all subjects (4). The latter study illustrates one of the main challenges in maintaining carnosinemia. Recent studies have explored targeting carnosinase, as low serum carnosinase activity, via polymorphism in the carnosine dipeptidase-1 (CNDP1) gene, protects patients with type 2 diabetes from diabetic nephropathy, and renal tissue carnosinase can be allosterically inhibited by administration of reduced glutathione (GSH), $\mathrm{N}$-acetylcysteine, and cysteine in diabetic mice 


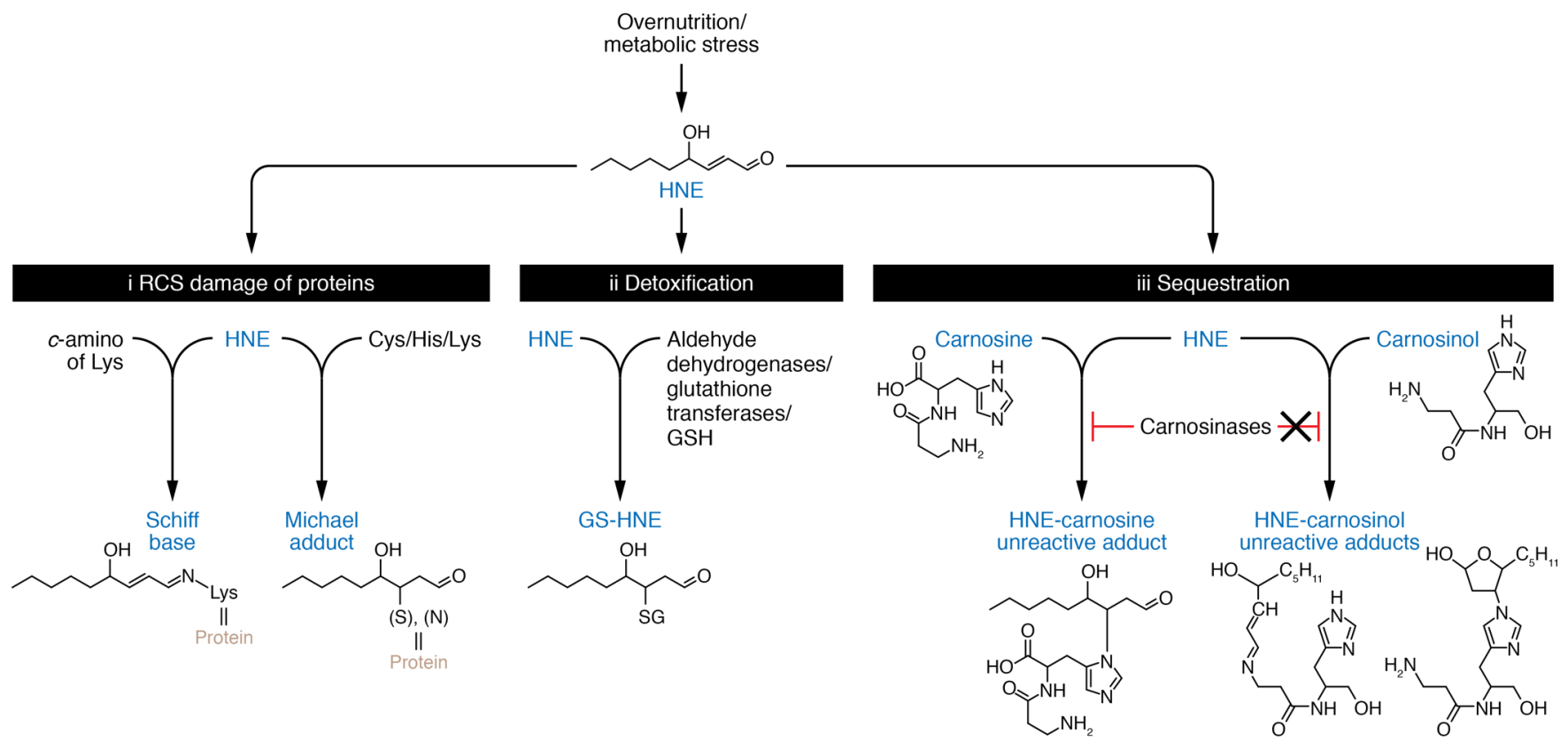

Figure 1. Fates of the reactive carbonyl HNE as a result of overnutrition and metabolic stress. (i) HNE is a RCS that damages proteins at various amino acid residues to form either a Schiff base or a Michael adduct. (ii) HNE can be detoxified through natural defense mechanisms including aldehyde dehydrogenases or glutathione transferases, which create a stable product that is exported out of cells and cleared by renal mechanisms. (iii) Left: HNE can also be sequestered by carnosine, creating a stable HNE-carnosine adduct that is nonreactive. However, carnosine is degraded by carnosinases, which limit HNE sequestration in vivo. Right: To circumvent carnosine degradation via carnosinases, a next-generation carnosine derivative called carnosinol was created, which is resistant to carnosinase degradation and has strong affinity for RSC.

(10). Further, the novel agent carnostatine (SAN9812) was shown to be a potent and selective carnosinase inhibitor in animal models (11). However, it is unclear whether this or related compounds are being pursued for use in humans. These findings highlight the need for innovative developments in carnosine and RCS scavenging to treat metabolic disorders of obesity.

\section{Evidence for the therapeutic potential of a novel carnosine derivative}

In the current issue, Anderson and colleagues have developed a novel carnosine derivative, carnosinol (2S)-20(3-amino propanoylamino)-3-(1H-imidazol-5-yl) propanol, which sequesters RCS but cannot be degraded by the naturally circulating carnosinase enzyme (12). The stated goal was to develop an RCS-sequestering agent stable in plasma that would be effective at scavenging circulating RCS and highly reactive toward damaging RCS. The obvious primary focus was ensuring stability of the agent in circulation. To achieve this goal, the group modified the carboxyl group of L-carnosine. The carboxyl group served as a sensible target, because while it is critical for carnosinase binding, in the current study it did not impact quenching and only slightly modified other chemical properties. Chemical analysis revealed that carnosinol had excellent quenching ability and enhanced lipophilic properties. The team then tested the ability of carnosinol to selectively bind and sequester both lipid-derived (HNE and ACR) and glucose-derived (methylglyoxal) aldehydes compared with L-carnosine and a number of other compounds known to scavenge RCS. Anderson and colleagues then moved to in vivo rodent studies by testing the effectiveness of a low and high dose of carnosinol to improve cardiometabolic risk factors and lower systemic inflammation and oxidative stress in rats fed a high-fructose diet through drinking water for a period of three weeks. They also tested whether carnosinol would improve similar outcomes in high-fat/high-sucrose (HFHS) diet-fed mice that were heterozygous (het) for glutathione peroxidase 4 (GPx4). GPx4 het mice are particularly susceptible to increased RCS, because GPX4 is obligatory for neutralizing lipid peroxides in cell membranes and circulating lipoproteins. In both fructose-fed rats and HFHS-fed GPx4 het mice, carnosinol showed a pronounced effect on improving a variety of factors including circulating insulin and glucose levels and liver inflammation and fibrosis. In mice, carnosinol improved ex vivo measures of glucose transport in skeletal muscle, validating tissue-specific effects. In both studies, these effects were associated with lowered lipid peroxidation measured by protein-HNE adducts. The authors presented additional measures including liquid chromatography-mass spectrometry (LC-MS) analysis of tissue free-carnosinol and carnosinol-aldehyde adducts to provide validation that carnosinol produced targeted effects associated with improved outcome measures. Finally, they also tested carnisonol reactivity in human serum, showing that carnisonol can form stable adducts with trace HNE in human serum.

\section{Considerations and future directions}

Despite the novel work presented by Anderson et al., these results should be interpreted with caution, as human clinical trials still need to be performed. Additionally, while Anderson and colleagues 
primarily focused on HNE biology, further studies are needed to examine the effects of carnosinol in mitigating the detrimental effects of other RCS such as glyoxal and methyglyoxal. Additional dose and duration studies are needed to carefully track whether carnosinol leads to reductions in oxidized stress followed by subsequent improvements in metabolism and inflammation. The current data, while impressive, do not definitively prove that carnosinol lowers oxidative stress followed by subsequent improvements and do not discount the possibility of insulin sensitivity and tissue lipid stores being altered through an alternative mechanism. Regardless, this compound is likely to serve as a powerful tool to test these links in the future.

The accumulation of HNE and other carbonyl species in tissues and the circulation imply that the natural defense system to detoxify these products has been compromised or outpaced by carbonyl flux. A common approach to dealing with this phenomenon has been to provide palliative therapy in the form of antioxidants and other bioactive compounds. Ristow and colleagues (13) showed that antioxidant supplementation impairs improvements in insulin sensitivity following exercise training through mitohormesis mechanisms, in which the natural antioxidant defense systems were downregulated as a result of supplementation. Thus, future studies must consider the adaptive effects of exogenous carnosinol supplementation on the natural antioxidant defense systems.

Another consideration is the increasing preponderance of PUFA-fortified foods available to consumers, given the putative beneficial effects of PUFAs in preventing cardiovascular disease. However, these
PUFA-fortified foods may contain oxidized products such as HNE as a result of the processing methods. Additionally, high, direct-heat cooking methods have also been shown to increase $\alpha, \beta$-unsaturated carbonyl formation as well as promote the formation of Maillard reaction products. Thus, studies examining the benefits of carnosinol in experimental models in which PUFAs and/or advanced glycation end-products are elevated in the diet are also warranted.

In summary, Anderson and colleagues have developed a novel carnosinol compound that is stable in the circulation, effectively binds HNE adducts, and has pronounced effects on lowering lipid peroxidation as well as on metabolic and inflammatory outcomes in vivo. Further studies are needed, but the potential for this compound to lower oxidative stress and partially mitigate the harmful effects of obesity and poor diet is exciting.

\section{Acknowledgments}

The authors are supported by NIH grants R01DK109948(toJH),1R01AR071263-01 (to JT), and R21AG056062-01 (to JT) and by a VA Merit Award (1I01BX002567-01, to JT).

Address correspondence to: John P. Thyfault, Department of Molecular and Integrative Physiology, University of Kansas Medical Center, 2067 Hemenway, 3901 Rainbow Blvd., Kansas City, Kansas 66160, USA. Phone: 913.588.1790; Email: jthyfault@kumc.edu.

1. Esterbauer H, Schaur RJ, Zollner H. Chemistry and biochemistry of 4-hydroxynonenal, malonaldehyde and related aldehydes. Free Radic Biol
Med. 1991;11(1):81-128.

2. Boldyrev AA, Aldini G, Derave W. Physiology and pathophysiology of carnosine. Physiol Rev. 2013;93(4):1803-1845.

3. Aldini G, Dalle-Donne I, Facino RM, Milzani A, Carini M. Intervention strategies to inhibit protein carbonylation by lipoxidation-derived reactive carbonyls. Med Res Rev. 2007;27(6):817-868

4. Regazzoni L, et al. A carnosine intervention study in overweight human volunteers: bioavailability and reactive carbonyl species sequestering effect. Sci Rep. 2016;6:27224.

5 . Yeum KJ, et al. Profiling histidine dipeptides in plasma and urine after ingesting beef, chicken or chicken broth in humans. Amino Acids. 2010;38(3):847-858.

6. Winstanley RP. Re: Holmes et al. Percutaneous osteosynthesis of the zygomatic buttress. $\mathrm{Br} \mathrm{J}$ Oral Maxillofac Surg 2001; 39: 286-288. Br J Oral Maxillofac Surg. 2002;40(1):86.

7. de Courten B, et al. Effects of carnosine supplementation on glucose metabolism: Pilot clinical trial. Obesity (Silver Spring). 2016;24(5):1027-1034.

8. Baye E, et al. Carnosine supplementation reduces plasma soluble transferrin receptor in healthy overweight or obese individuals: a pilot randomised trial [published online ahead of print August 22, 2018]. Amino Acids. https://doi. org/10.1007/s00726-018-2623-6.

9. Baye E, et al. Effect of carnosine supplementation on the plasma lipidome in overweight and obese adults: a pilot randomised controlled trial. Sci Rep. 2017;7(1):17458.

10. Peters V, et al. Allosteric inhibition of carnosinase (CN1) by inducing a conformational shift. JEnzyme Inhib Med Chem . 2017;32(1):1102-1110.

11. Qiu J, et al. Identification and characterisation of carnostatine (SAN9812), a potent and selective carnosinase (CN1) inhibitor with in vivo activity [published online ahead of print June 22, 2018]. Amino Acids. https://doi.org/10.1007/s00726018-2601-z.

12. Anderson EJ, et al. A carnosine analog mitigates metabolic disorders of obesity by reducing carbonyl stress. J Clin Invest. 2018;128(12):5280-5293.

13. Ristow M, et al. Antioxidants prevent health-promoting effects of physical exercise in humans. Proc Natl Acad Sci U S A. 2009;106(21):8665-8670. 